\title{
HIGHLIGHTS
}

GENETICS

\section{Potential causal variants for RA identified}

Genome-wide association studies (GWASs) have previously revealed

Raychaudhuri and colleagues narrowed down the possible genetic associations to six potentially causal variants for RA
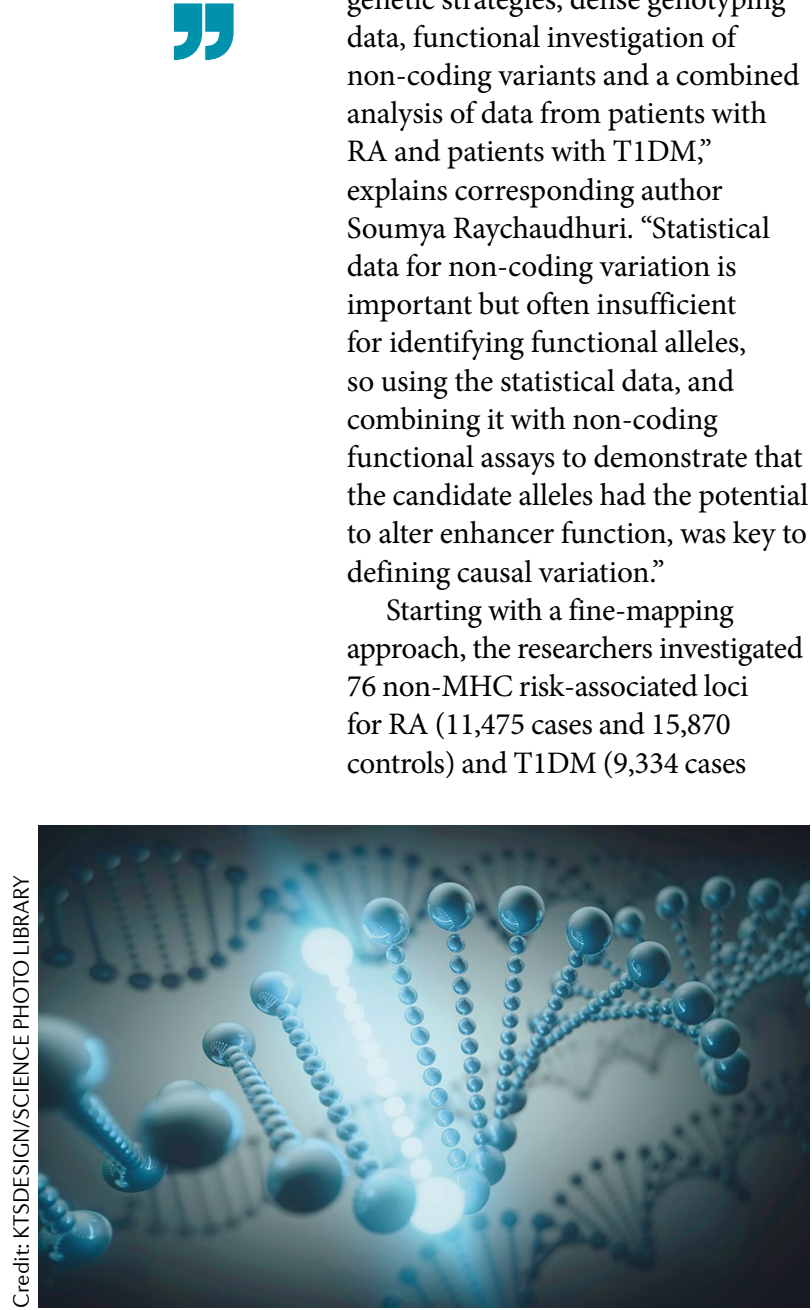

and 11,111 controls). The resulting list was refined to include only loci with $\leq 10$ variants in their credible sets, and these loci were taken forward for functional studies (electrophoretic mobility shift assays and allele-specific luciferase assays) using immortalized $\mathrm{T}$ cell and monocyte lines.

Using this integrated approach, Raychaudhuri and colleagues narrowed down the possible genetic associations to six potentially causal variants for RA. These variants included missense mutations in TYK2 (rs35018800) and DNASE1L3 (rs35677470), an indel in TNFAIP3 (rs35926684) and a non-coding mutation in the CD28-CTLA4 locus (rs117701653). Shared missense mutations with T1DM in PTPN22 and TYK2 that had been previously described in GWASs were also confirmed.

"Amongst the most exciting results are a loss-of-function allele in DNASE1L3 and non-coding causal alleles in TNFAIP3 and CD28CTLA4," says Raychaudhuri. "These results point to key pathways and genes that are driving RA risk."

The loss-of-function missense mutation in DNASE1L3 produced a nuclease that could no longer cleave DNA during apoptosis. Importantly, similar missense mutations in DNASE1L3 have previously been reported for systemic sclerosis and systemic lupus erythematosus (SLE).

Similarly, variants of TNFAIP3 are associated with several autoimmune diseases, including SLE and Sjögren syndrome. A20, the protein coded for by TNFAIP3, reduces signalling via the transcription factor NF- $\kappa \mathrm{B}$ and thereby inhibits apoptosis. Interestingly, the results of $\mathrm{Hi}-\mathrm{C}$ promoter-capture analysis suggested that the rs35926684 region interacts with the promoters for TNFAIP3, IL22RA (involved in inflammation, apoptosis and angiogenesis) and IFNGR1 (involved in interferon responses), indicating that several genes could be influenced by this one variant.

The rs 117701653 variant of CD28-CTLA4 (located in the CD28 region) also showed interactions with multiple promoters via $\mathrm{Hi}-\mathrm{C}$ analysis, suggesting that this variant might affect CTLA4 (involved in $T$ cell co-stimulation) and RAPH1 (involved in cell adhesion).

"The success of this study is to identify primary disease-associated variants," says Gerry Wilson, an expert in the genetics that underlie RA who was not involved in this study. "It's a great example of integration of state-of-the-art statistical analysis and straightforward biological experiments. Moving forward, it will be important to determine if the functional effects observed are specific to T cells or potentially occur in other relevant cell types such as synovial fibroblasts or B cells."

"There remain questions as to what context these alleles are working in, both in terms of cell-type and cell-state, what the downstream genes are that are regulated by the RA risk alleles and what the upstream regulatory machineries are that are driving RA risk," concludes Raychaudhuri. "Additional cellular investigations including CRISPR strategies and single cell investigation of inflamed tissues in RA will be critical to define the set of candidate genes driving cellular phenotypes."

Joanna Collison

ORIGINAL ARTICLE Westra, H.--. et al.

Fine-mapping and functional studies highlight potential causal variants for rheumatoid arthritis and type 1 diabetes. Nat. Genet. 50, 1366-1374 (2018) 